\title{
Angular Dependence of Spin Torque Critical Currents for CPP-GMR Read Heads
}

\author{
Neil Smith, J. .A. Katine, Jeffrey R. Childress, and Matthew J. Carey,
}

\begin{abstract}
This paper employs (analytical) micromagnetic modeling to derive expressions for the critical current at the onset of spin-transfer-torque (STT) instability in CPP-GMR read heads, as a function of the relative angle between the free and reference layer magnetizations, including a general angular dependent STT coefficient. Experimental measurement of the angular dependence of the critical currents are made on 50-nm sized CPP-GMR devices with synthetic antiferromagnet pinned layers, and fabricated using e-beam lithography. The results are consistent with prior theoretical models, but indicate perhaps unanticipated implications for read head operation.
\end{abstract}

Index Terms-current-perpendicular-to-plane, giant magnetoresistance (GMR), read head, spin-torque.

\section{INTRODUCTION}

Qince first described theoretically [1]-[2], the phenomenon $N_{\text {of }}$ spin-transfer-torques (STT) from spin-polarized conduction electrons passing current perpendicular-to-plane (CPP) through thin ferromagnetic films has received much recent attention, including theoretical [3]-[5], modeling [6][12], and experimental [11]-[18]. This interest in STT has been mostly as a field-independent means for magnetization reversal in thin magnetic films, particularly that of the free layer in MRAM memory elements. Here, the STT-induced reversal process is between two uniaxial stable states with free and reference layer magnetizations $\hat{\boldsymbol{m}}_{\text {free }}$ and $\hat{\boldsymbol{m}}_{\text {ref }}$ being collinear. Receiving less attention is the critical current for the onset of undesirable STT-induced noise/oscillations of the free layer in CPP-GMR spin-valve recording heads [19]-[20], where $\hat{\boldsymbol{m}}_{\text {free }}$ has unidirectional stability and is orthogonal to $\hat{\boldsymbol{m}}_{\text {ref }}$ in the quiescent state. Receiving relatively little experimental [21] or modeling consideration is the influence here of any intrinsic angular (i.e., $\hat{\boldsymbol{m}}_{\text {free }} \cdot \hat{\boldsymbol{m}}_{\text {ref }}$ ) dependence of STT, which was first discussed theoretically by Slonczewski [1]. This paper will first discuss theoretical modeling of the critical currents, and then describe a set of detailed experimental measurements of the angular dependence of these critical currents in 50-nm sized CPP-GMR devices.

Manuscript received February 7, 2005

The authors are with Hitachi Global Storage Technologies, San Jose, CA 95120 USA (phone: 408-323-7294, e-mail: neil.smith@ hitachigst.com.

\section{STABILITY ANALYSIS}

The results below are based on the simple model of a CPP spin-valve sensor illustrated in Fig. 1. Each magnetic layer is treated as a uniformly magnetized macro-spin with a unit magnetization vector, $\hat{\boldsymbol{m}} \equiv\left(m_{x}, m_{y}, m_{z}\right)$. The magnetization $\hat{\boldsymbol{m}}_{\text {pin }}$ of the bottom "pinned" layer is assumed to be exchange pinned along the $+\hat{z}$-axis by an adjacent antiferromagnetic (AF) layer (not shown), and is also strongly AF-coupled to $\hat{\boldsymbol{m}}_{\text {ref }}$ of the "reference" layer through a thin Ru spacer. Both $\hat{\boldsymbol{m}}_{\text {pin }}$ and $\hat{\boldsymbol{m}}_{\text {ref }}$ are assumed rigidly fixed. The free layer magnetization $\hat{\boldsymbol{m}}_{\text {free }}(t)$ is treated as a dynamical variable.

In the absence of STT effects, the equations of motion for $\hat{\boldsymbol{m}}_{\text {free }}(t)$ are taken to be the standard Gilbert equations:

$$
\begin{gathered}
d \hat{\boldsymbol{m}} / d t=\gamma\left(\boldsymbol{H}_{\mathrm{eff}} \times \hat{\boldsymbol{m}}\right)+\alpha(\hat{\boldsymbol{m}} \times d \hat{\boldsymbol{m}} / d t) \\
\Rightarrow(\hat{\boldsymbol{m}} \times d \hat{\boldsymbol{m}} / d t)+\alpha d \hat{\boldsymbol{m}} / d t=\gamma\left[\boldsymbol{H}_{\mathrm{eff}}-\left(\boldsymbol{H}_{\mathrm{eff}} \cdot \hat{\boldsymbol{m}}\right) \hat{\boldsymbol{m}}\right] \\
\boldsymbol{H}_{\mathrm{eff}} \equiv-\left(1 / M_{s} V_{\text {free }}\right) \partial E_{\text {free }} / \partial \hat{\boldsymbol{m}}
\end{gathered}
$$

using $\hat{\boldsymbol{m}} \leftrightarrow \hat{\boldsymbol{m}}_{\text {free }}$ interchangeably as clarity permits. $M_{s}$, $V_{\text {free }}$ and $E_{\text {free }}(\hat{\boldsymbol{m}})$ are the saturation magnetization, volume and free energy of the free layer, respectively, $\gamma>0$ is the gyromagnetic ratio, and $\alpha$ is the Gilbert damping parameter.

The physical basis for STT has been described earlier by Slonczewski [1]. From a modeling perspective, the effect of STT on the motion of $\hat{\boldsymbol{m}}_{\text {free }}(t)$ was found in [1] to result in an

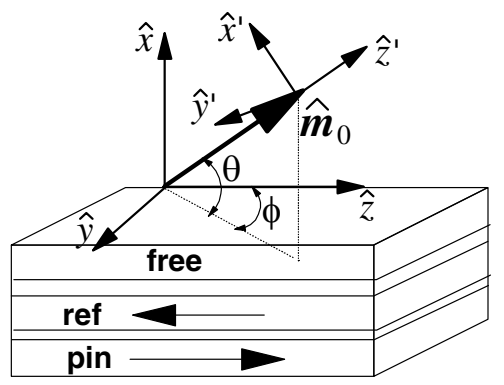

Fig. 1 Cartoon of magnetic layers of CPP-GMR device. Positive electron current $I_{e}>0$ is defined when electrons travel along the $+\hat{\boldsymbol{x}}$ axis. For the rotated $x^{\prime} y^{\prime} z^{\prime}$ coordinate system, the $y^{\prime}$ axis lies in the $y-z$ plane. 
added spin-torque contribution to $d \hat{\boldsymbol{m}} / d t$ of the form:

$$
\begin{gathered}
(d \hat{\boldsymbol{m}} / d t)_{\mathrm{st}} \equiv \beta\left(\hat{\boldsymbol{m}}_{\text {free }} \cdot \hat{\boldsymbol{m}}_{\mathrm{ref}}\right) \gamma H_{\mathrm{st}} \hat{\boldsymbol{m}}_{\text {free }} \times\left(\hat{\boldsymbol{m}}_{\mathrm{ref}} \times \hat{\boldsymbol{m}}_{\text {free }}\right) \\
H_{\mathrm{st}} \equiv(\gamma \hbar / 2 e) P I_{e} /\left(M_{s} V_{\text {free }}\right)
\end{gathered}
$$

where $P$ is the polarization of the conduction electrons, and electron current $I_{e}$ is taken as positive when electrons flow from reference layer to free layer (along $+\hat{\boldsymbol{x}}$-axis in Fig. 1). The dimensionless factor $\beta\left(\hat{\boldsymbol{m}}_{\text {free }} \cdot \hat{\boldsymbol{m}}_{\text {ref }}\right)>0$ is of order unity, with a predicted theoretical form described earlier [1]. It will be treated here in general [10], leaving its angular dependence to be the primary subject of the work of this paper.

Adding the $d \hat{\boldsymbol{m}} / d t$ contribution of (2) to (1), the form of the Gilbert equations including STT is unchanged, provided one simply redefines the effective field $\boldsymbol{H}_{\text {eff }}$ as:

$$
\boldsymbol{H}_{\text {eff }}=-\left(1 / M_{s} V_{\text {free }}\right) \partial E_{\text {free }} / \partial \hat{\boldsymbol{m}}-\beta(\hat{\boldsymbol{m}}) H_{\text {st }} \hat{\boldsymbol{m}}_{\text {ref }} \times \hat{\boldsymbol{m}}_{\text {free }}
$$

The equations of motion can be transformed into a (primed) coordinate system (Fig. 1) where $\hat{z}^{\prime} \equiv \hat{\boldsymbol{m}}\left(t=t_{0}\right)$ is parallel to $\hat{\boldsymbol{m}}_{0} \equiv \hat{\boldsymbol{m}}_{\text {free }}\left(t_{0}\right)$ at time $t_{0}$. The equations hold for $t \geq t_{0}$ when the $t w o$-dimensional vector $\boldsymbol{m}^{\prime} \equiv\left(m_{x}^{\prime}, m_{y}^{\prime}\right)$ is small, i.e., $\left|\boldsymbol{m}^{\prime}\right| \perp 1$. The transformation is described by a $3 \times 2$ matrix $\vec{R}$ (see Appendix) such that $\hat{\boldsymbol{m}}=\hat{\boldsymbol{m}}_{0}+\vec{R} \cdot \boldsymbol{m}^{\prime}$ to first order in $\boldsymbol{m}^{\prime}(t)$. The linearized equations of motion for $\boldsymbol{m}^{\prime}(t)$ are

$$
\begin{aligned}
& \frac{1}{\gamma} \frac{d}{d t}\left(\begin{array}{cc}
\alpha & -1 \\
1 & \alpha
\end{array}\right) \cdot\left(\begin{array}{l}
m_{x}^{\prime} \\
m_{y}^{\prime}
\end{array}\right)+\overrightarrow{\boldsymbol{H}}^{\prime} \cdot \boldsymbol{m}^{\prime}(t)=\vec{R}^{\top} \cdot \boldsymbol{H}_{\mathrm{eff}}\left(\hat{\boldsymbol{m}}_{0}\right) \\
& \overrightarrow{\boldsymbol{H}}^{\prime} \equiv \vec{R}^{\top} \cdot \overrightarrow{\boldsymbol{H}} \cdot \vec{R},\left.\overrightarrow{\boldsymbol{H}} \equiv\left[\left(\hat{\boldsymbol{m}} \cdot \boldsymbol{H}_{\mathrm{eff}}\right) \overrightarrow{\mathbf{1}}-\left(\partial \boldsymbol{H}_{\mathrm{eff}} / \partial \hat{\boldsymbol{m}}\right)\right]\right|_{\hat{\boldsymbol{m}}=\hat{\boldsymbol{m}}_{0}}
\end{aligned}
$$

where $\overrightarrow{\boldsymbol{H}}$ and $\overrightarrow{\boldsymbol{H}}^{\prime}$ are $3 \times 3$ and $2 \times 2$ Cartesian tensors, $\vec{R}^{\top}$ is the $2 \times 3$ transpose of $\vec{R}$, and $\overrightarrow{\mathbf{1}}$ is the $3 \times 3$ identity tensor.

The present interest is in the prediction of the onset of STTinduced dynamical instability at critical current $I_{e} \rightarrow I_{e}^{\text {crit }}$, and in particular, the angular $(\theta, \phi)$ dependence of $I_{e}^{\text {crit }}$ due to that of $\beta\left(\hat{\boldsymbol{m}}_{\text {free }} \cdot \hat{\boldsymbol{m}}_{\text {ref }}\right)$. The free-layer reference vector $\hat{\boldsymbol{m}}_{0}$ is assumed to be an equilibrium for $\left|I_{e}\right|<\left|I_{e}^{\text {crit }}\right|$ satisfying $\vec{R}^{\top} \cdot \boldsymbol{H}_{\text {eff }}\left(\hat{\boldsymbol{m}}_{0}\right)=0$. Stability of an equilibrium $\hat{\boldsymbol{m}}_{0}$ requires natural-mode solutions $\boldsymbol{m}^{\prime} \propto e^{-i \lambda t}$ of (4) with $\operatorname{Im} \lambda<0$, which leads to following two necessary stability conditions [10]:

$$
\begin{aligned}
& \operatorname{det} \overrightarrow{\boldsymbol{H}}^{\prime} \equiv H_{x x}^{\prime} H_{y y}^{\prime}-H_{x y}^{\prime} H_{y x}^{\prime}>0 \\
& \alpha\left(H_{x x}^{\prime}+H_{y y}^{\prime}\right)+\left(H_{y x}^{\prime}-H_{x y}^{\prime}\right)>0
\end{aligned}
$$

In the absence of STT $\left(H_{\text {st }}=0\right)$, both $\overrightarrow{\boldsymbol{H}}$ and $\overrightarrow{\boldsymbol{H}}^{\prime}$ are symmetric, and (5) reduces to $\operatorname{det} \overrightarrow{\boldsymbol{H}}^{\prime}>0$ ( $\overrightarrow{\boldsymbol{H}}^{\prime}$ positivedefinite). However, when $H_{\text {st }} \neq 0$, the $\hat{\boldsymbol{m}}_{\text {ref }} \times \hat{\boldsymbol{m}}_{\text {free }}$ term in (3) yields a resultant $\overrightarrow{\boldsymbol{H}}^{\prime}$ that is nonsymmetric $\left(H_{y x}^{\prime} \neq H_{x y}^{\prime}\right)$. As detailed elsewhere [10], the physical consequences of this nonsymmetry can lead to a systematic transfer of energy from the current $I_{e}$ to the macro-spin $\hat{\boldsymbol{m}}_{\text {free }}$, resulting in an STTinduced instability described by the second result of (5).

A simplified model corresponding to the experiment described in Sec. (III) is that of a free-layer with in-plane unidirectional anisotropy provided by an in-plane ( $y-z$ plane in Fig. 1) magnetic field $\boldsymbol{H}_{\mathrm{p}}$, and a uniaxial out-of-plane (shape) anisotropy $H_{\perp}\left(\sim 4 \pi M_{s}-H_{k \perp}\right)$. The reference layer $\hat{\boldsymbol{m}}_{\text {ref }}$ is also assumed to be in-plane. The derivation steps and linear algebra necessary to evaluate (5) for this case are outlined in the Appendix. The second stability condition of (5) becomes:

$$
\begin{gathered}
\left(2 H_{\mathrm{p}}+H_{\perp}\right) \alpha+H_{\mathrm{st}}\left[2 q \beta(q)-\left(1-q^{2}\right) \beta^{\prime}(q)\right]>0 \\
H_{\mathrm{p}} \equiv\left|\boldsymbol{H}_{\mathrm{p}}\right|, q \equiv \hat{\boldsymbol{m}}_{0} \cdot \hat{\boldsymbol{m}}_{\mathrm{ref}}, \beta^{\prime} \equiv d \beta / d q
\end{gathered}
$$

Unlike the more commonly considered collinear case $|q|=1$, STT-stability is, in general, influenced by both the function $\beta(q)$ and its derivative $d \beta / d q$. This is particularly true in the quasi-orthogonal case $\left|q=\hat{\boldsymbol{m}}_{0} \cdot \hat{\boldsymbol{m}}_{\text {ref }}\right|<<1$ that is relevant to the desired nominal bias state of CPP-GMR read heads.

The value of $H_{\mathrm{st}}\left(I_{e}\right)$ (see (2)) for which the left side of (6) just vanishes determines the critical current $I_{e}^{\text {crit }}(q)$. By taking the ratio of (6) evaluated at $I_{e}^{\text {crit }}(q)$ and $I_{e}^{\text {crit }}(q=-1)$, the resultant quotient may be arranged in the form:

$$
\begin{gathered}
\frac{1-q^{2}}{2} \frac{d \rho}{d q}-q \rho(q)=F(q) \equiv \frac{\left.\left(2 H_{\mathrm{p}}+H_{\perp}\right)\right|_{q}}{\left.\left(2 H_{\mathrm{p}}+H_{\perp}\right)\right|_{-1}} \frac{I_{e}^{\text {crit }}(-1)}{I_{e}^{\text {crit }}(q)} \\
\rho(q) \equiv \beta(q) / \beta(-1)
\end{gathered}
$$

The result in (7) eliminates physical parameters $\alpha, P$, and $V_{\text {free }}$, and contains only quantities that are subject to experimental measurement (Sec. IIIB). Since $H_{\perp}>>\left|\boldsymbol{H}_{\mathrm{p}}\right|$ in practical cases, the dependence of $q\left(\boldsymbol{H}_{\mathrm{p}}\right)$ implicit in (7) need not be known to great accuracy. A dual branch solution of the differential equation in (7), chosen to avoid integration across a zero of $F(q)$ (infinity/discontinuity in $I_{e}^{\text {crit }}(q)$ ) is given by

$$
\beta^{ \pm}(q) / \beta(-1)=2 /\left(1-q^{2}\right) \int_{\mp 1}^{q} F\left(q^{\prime}\right) d q^{\prime}
$$

Using (7) and/or (8) requires measuring $I_{e}^{\text {crit }}$ as a function of $q=\hat{\boldsymbol{m}}_{\text {free }} \cdot \hat{\boldsymbol{m}}_{\text {ref }}$, which will be discussed further in Sec. III. 


\section{EXPERIMENT}

\section{A. Film Deposition and Device Fabrication}

Samples were fabricated by magnetron sputtering at room temperature on silicon. The base pressure was $2 \times 10-8$ Torr. Ar pressure during deposition was $2 \mathrm{mT}$. The film stack consists of (in nanometers): a bottom lead structure of: $\mathrm{Ta}(5) / \mathrm{Cu}(40) / \mathrm{Ta}(2)$, followed by the spin-valve: $\mathrm{PtMn}(15) / \mathrm{Co}_{84} \mathrm{Fe}_{16}(2) / \mathrm{Ru}(0.8) / \mathrm{Co}_{84} \mathrm{Fe}_{16}(2.2) / \mathrm{Cu}(3.5) / \mathrm{Co}_{84} \mathrm{Fe}_{16}$ (1) $/ \mathrm{Ni}_{88} \mathrm{Fe}_{12}(2.4)$, and finally a top cap of $\mathrm{Cu}(20) / \mathrm{Ru}(4) / \mathrm{Ta}$ (2.5). The thick $\mathrm{Cu}$ cap protects the free layer from oxidation during the annealing process, and allows good electrical contact to be made between the top lead and the pillar. The $\mathrm{CoFe} / \mathrm{Ru} / \mathrm{CoFe}$ synthetic-antiferromagnet (SAF) substantially reduces the dipole field on the free layer, facilitating STT measurements with small in-plane fields. The PtMn-SAF exchange coupling keeps the $2.2 \mathrm{~nm}$-thick $\mathrm{CoFe}$ reference layer well pinned for collinear in-plane fields up to $\sim 2 \mathrm{kOe}$.

Following film deposition, electron beam lithography was used to pattern a high resolution negative e-beam resist, HSQ. The Fox-12 HSQ formulation (available from Dow Corning) was spun to a thickness of $100 \mathrm{~nm}$. Electron beam exposure essentially converts HSQ into $\mathrm{SiO}_{2}$, whose high resistance to ion milling allows the resist to directly serve as a high-fidelity mask during the etching of our devices. After milling, $100 \mathrm{~nm}$ of aluminum oxide was ion-beam deposited onto the wafer, encapsulating the milled pillar and resist. Due to the mechanical instability of the resist mask, the HSQ on top of the pillar is readily removed during a short chemical mechanical polish (CMP), creating a via in the aluminum oxide that allows self-aligned contact to the top of the pillar. To minimize contact resistance, it is important to perform an in situ sputter etch prior to depositing the top $\mathrm{Cr} / \mathrm{Au}$ lead.

\section{B. Measurement of Critical Currents}

Fig. 2 shows $\delta R$ vs. applied field $\boldsymbol{H}_{\text {a }}$ for a $50 \mathrm{~nm}$ diameter circular pillar CPP-GMR device $(\approx 35 \Omega)$, measured on a Monarch prober. The $\delta R=0$ point for the $\delta R-H_{\mathrm{a} z}$ reference loop is chosen at $H_{\mathrm{az}}=-1 \mathrm{kOe}$ (low-resistance state $\left.\hat{\boldsymbol{m}}_{\text {free }} \cong \hat{\boldsymbol{m}}_{\text {ref }} \cong-\hat{\boldsymbol{z}}\right)$. To compensate for $I_{e}$-dependent thermal shift, the $\delta R-H_{\text {ay }}$ curves with $I_{e}>0$ are individually zeroshifted to coalesce at large $\left|H_{a y}\right|$ with the $I_{e}=-0.25 \mathrm{~mA}$ $\delta R-H_{\text {ay }}$ loop, which is further aligned at $\boldsymbol{H}_{\mathrm{a}} \cong 0$ with the $\delta R-H_{\mathrm{a} z}$ loop. The latter is non-hysteretic, but indicates an internal field $\boldsymbol{H}_{\mathrm{i}} \approx+160 \hat{z}$ Oe due to residual magnetostatic coupling between free and ref/pin layers, yielding a highresistance remnant state $\left(\hat{\boldsymbol{m}}_{\text {free }} \cong \hat{z}, \hat{\boldsymbol{m}}_{\text {ref }} \cong-\hat{z}\right)$ at $\boldsymbol{H}_{\mathrm{a}}=0$.

Although qualitatively similar with others, this device was chosen for its greater than typical symmetry about $H_{\mathrm{ay}}=0$ in its $\delta R-H_{\mathrm{a} y}$ loops, as expected from the model of Fig. 1.

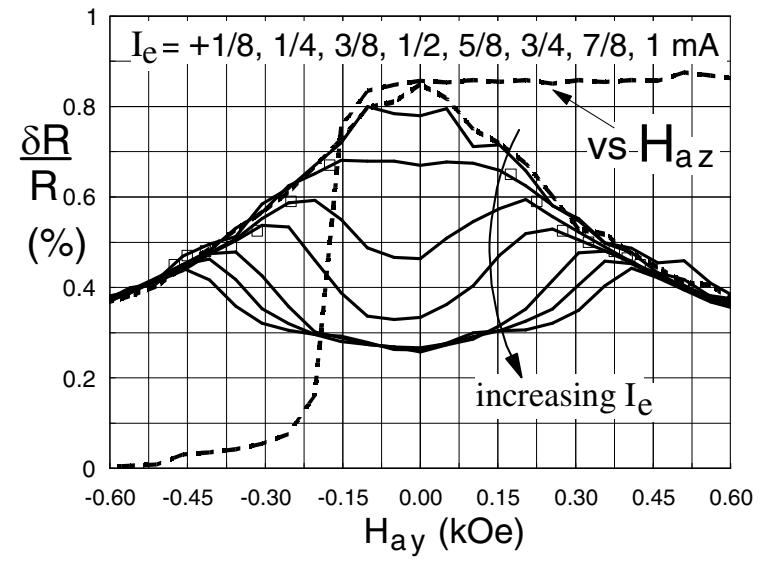

Fig. $2 \delta R-H_{\text {ay }}$ loops for various $I_{e}>0$ as indicated. The Dashed curves show $\delta R-H_{\text {ay }}$ and $\delta R-H_{\text {az }}$ reference loops at $I_{e}=-0.25 \mathrm{~mA}$. The open square symbols indicate estimated critical points.

Noteworthy here is the gross distortion in the $\delta R-H_{\mathrm{a} y}$ loops, resulting in minima at $H_{a y} \approx 0$ which grow broader and deeper with increasing positive $I_{e}$. By contrast, $\delta R-H_{\mathrm{a} y}$ loops for $-1 \mathrm{~mA} \leq I_{e}$ resemble the $-0.25 \mathrm{~mA}$ loop (Fig. 2).

Fig. 3 shows $1-\mathrm{MHz}$ bandwidth rms power spectral density vs. $I_{e}$, measured at $75 \mathrm{MHz}$. on a high-frequency prober using an Agilent-E4440A spectrum analyzer. The latter is sweeptriggered by the function generator that drives a $2-\mathrm{Hz}$ sawtooth current into the CPP-GMR device. Subtracting out the $I_{e}=0$ electronics noise leaves a residual $\approx 0.15(\mathrm{nV} / \sqrt{\mathrm{Hz}}) / \mathrm{mA}$ magnetic/thermal background. Of more significance here is the much larger, telegraph-like noise (as revealed by the full spectrum) which onsets sharply for positive, $H_{\mathrm{a} y}$-dependent "critical" values of $I_{e}$. Combined, Figs. 2 and 3 strongly suggest the STT-induced critical instability of the unidirectional device modeled in Sec. II. In particular, the uniquely stable state $\hat{\boldsymbol{m}}_{\text {ref }} \cong-\hat{\boldsymbol{z}}$, and $\hat{\boldsymbol{m}}_{\text {free }} \approx \hat{\boldsymbol{H}}_{\mathrm{p}}$ becomes

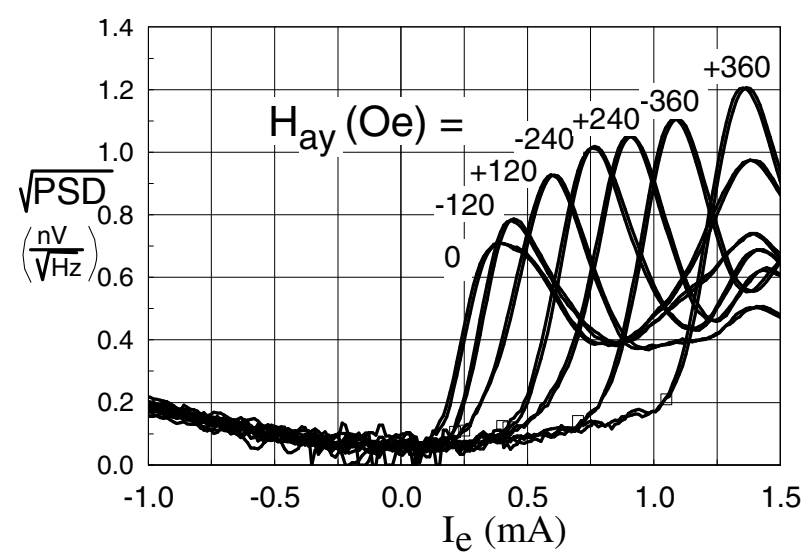

Fig. 3 rms power spectral density (PSD) at $75 \mathrm{MHz}$ vs $I_{e}$, for various values of $H_{\mathrm{ay}}$ as indicated. The open square symbols indicate the estimated critical points. All curves are averaged over $\sim 30$ cycles. 


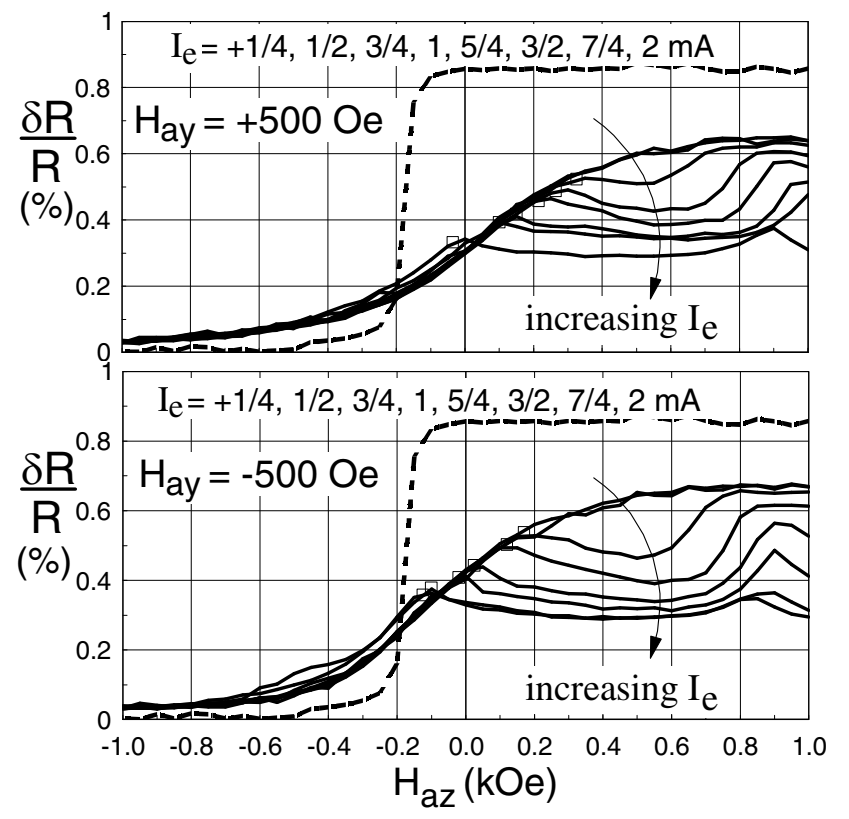

Fig. $4 \delta R-H_{\mathrm{a} z}$ curves with fixed $H_{\mathrm{a} y}= \pm 500 \mathrm{Oe}$ (as labeled), for various positive values of $I_{e}$. The open square symbols indicate the estimated critical points. The dashed $\delta R-H_{\mathrm{a} z}$ curve is from Fig. 2 .

unstable when $I_{e}>I_{e}^{\text {crit }}>0$, after which $\hat{\boldsymbol{m}}_{\text {free }}(t)$ undergoes a continual quasi-chaotic motion indicated by the large-scale noise in Fig. 3. The resultant reduction in the time-averaged value $\left\langle\hat{\boldsymbol{m}}_{\text {free }} \cdot \hat{z}\right\rangle \perp 1$ lowers the apparent resistance in $\delta R-H_{\mathrm{a} y}$ loops of Fig. 2. Comparing Figs. 2-3 shows fairly good agreement in the estimation of the "critical points" $\left(I_{e}^{\text {crit }}, H_{\mathrm{ay}}^{\text {crit }}\right)$ as determined by the rapid onset of RF noise, or the somewhat less distinctive onset of negative $\delta R$ deviations from the stable $\delta R-H_{\mathrm{ay}}$ loop shape with $I_{e}=-0.25 \mathrm{~mA}$. (Critical point evaluation here is somewhat subjective at much beyond $\sim 10 \%$ precision.) Figs. 2-3 also indicate some residual $\pm H_{\mathrm{a} y}$ asymmetry in $I_{e}^{\text {crit }}\left(H_{\mathrm{a} y}\right)$ (e.g., \pm 360 Oe in Fig. 3 ).

To simulate the bias conditions for an operating read head, $\delta R-H_{\mathrm{a} z}$ loops are measured with a fixed $H_{\mathrm{a} y}= \pm 500$ Oe to approach the nominal bias state $\hat{\boldsymbol{m}}_{\text {ref }} \cdot \hat{\boldsymbol{m}}_{\text {free }} \approx 0$, linearizing the response of the device to $z$-axis fields. These loops, for either polarity of $I_{e}$ are shown in Figs. 4 and 5. Similar to Fig. 2 , these $\delta R$ curves are zero-shifted to coalesce at large positive or negative $\left|H_{a z}\right|$, respectively, and such that the undistorted loops for $\left|I_{e}\right| \leq 0.5 \mathrm{~mA}$ yield the "expected" $\delta R$ value (see Sec. IV) at $H_{\mathrm{a} z}=-1 \mathrm{kOe}$ relative to the reference $\delta R-H_{\mathrm{a} z}$ loop. This procedure independently achieves fairly consistent values of $\delta R / R \approx 0.4 \%$ at $\left(\left|H_{\mathrm{ay}}\right|=500 \mathrm{Oe}, H_{\mathrm{az}}=0\right)$ when comparing the four sets of data of Figs. 4-5 with that of Fig. 2. In Figs. 4-5, the now bipolar (positive in Fig. 4, negative in

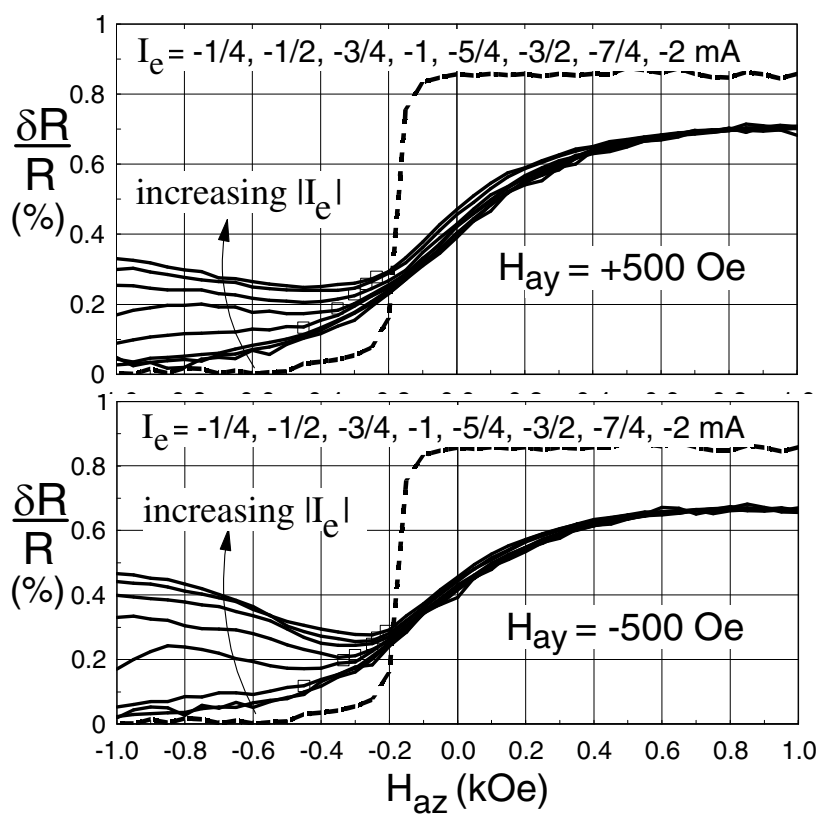

Fig. $5 \delta R-H_{\mathrm{a} z}$ curves with fixed $H_{\mathrm{a} y}= \pm 500$ Oe (as labeled), for various negative values of $I_{e}$. The open square symbols indicate the estimated critical points. The dashed $\delta R-H_{\mathrm{a} z}$ curve is from Fig. 2 .

Fig. 5) critical currents $\left|I_{e}^{\text {crit }}\left(\boldsymbol{H}_{\mathrm{a}}\right)\right|>0.5 \mathrm{~mA}$ can be estimated from the $\left(I_{e}, \boldsymbol{H}_{\mathrm{a}}\right)$ locations of the initial $\delta R$-deviations from the $\left|I_{e}\right| \leq 0.5 \mathrm{~mA}$ loops. As before, these data show some residual $\pm H_{\text {ay }}$ asymmetry. Measuring $\left(I_{e}^{\text {crit }}, \boldsymbol{H}_{a}^{\text {crit }}\right)$ here using the PSD required a two-axis field capability that was not available on the high frequency tester at the time of this study.

Fig. 6 illustrates the determination of the critical currents $I_{e}^{\text {crit }}\left(q \equiv \hat{\boldsymbol{m}}_{\text {fre }} \cdot \hat{\boldsymbol{m}}_{\text {ref }}= \pm 1\right) \quad$ PSD measurements. The total $z-$ axis field on the free layer is $H_{\mathrm{p} z} \cong H_{\mathrm{a} z}+H_{\mathrm{i}}$, with $H_{\mathrm{i}} \approx$ +160 Oe. Though the small difference in apparent $I_{e}^{\text {crit }}$ by inspection of the $H_{\mathrm{az}}=+180$ and +500 Oe loops $\left(H_{\mathrm{p} z}=\right.$ $+340,+660$ Oe) is consistent with Eq. (6), the larger difference between the $H_{\mathrm{a} z}=0$ and +180 Oe loops, and the gross

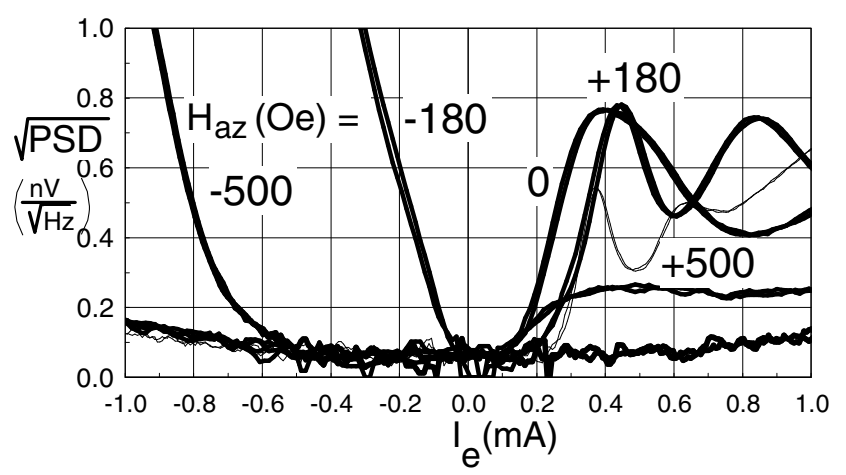

Fig. $6 \mathrm{PSD}$ at $75 \mathrm{MHz}$ vs $I_{e}$. at $H_{\mathrm{ay}}=0, H_{\mathrm{az}}$ as indicated. The finelined curve is for $H_{\mathrm{a} z}=+500$ Oe. All curves are averaged over $\sim 30$ cycles. 
difference between the $H_{\mathrm{a} z}=-500$ and 0 Oe loops $\left(H_{\mathrm{p} z}=-\right.$ 340 and -20 Oe, the latter being magnetothermally metastable) cannot be so accounted for. They are instead due to thermal fluctuations over $\sim 0.1 \mathrm{sec}$ measurement times [18]. As these data suggest thermal effects are small for $\left|\boldsymbol{H}_{\mathrm{p}}\right| \geq 300$ Oe, the values $I_{e}^{\text {crit }}(q=-1) \cong+0.2 \mathrm{~mA}$, and $I_{e}^{\text {crit }}(q=+1) \cong-0.6 \mathrm{~mA}$ (at $\left|\boldsymbol{H}_{\mathrm{p}}\right| \cong 340$ Oe ) will be used for the analysis in Sec. IV.

\section{ANALYSIS AND DISCUSSION}

Both original [1] and later [5] theoretical formulations of STT by Slonczewski predict the following form [22] for $\beta(q)$ :

$$
\beta(q) / \beta(-1)=(1-A) /[1+A q]
$$

For the present CPP-GMR film stack, the estimate $A \cong 0.5$ for the constant $A$ follows from the measurements of Fig. 6, and the relationship $\beta(+1) / \beta(-1)=I_{e}^{\text {crit }}(-1) / I_{e}^{\text {crit }}(+1) \cong 1 / 3$ from (7). However, theory ([5], [23]) also predicts a direct relation between the $q$-dependence of $\beta$ (i.e., $A \neq 0$ ) and a nonlinear q-dependence for the CPP-GMR resistance variation :

$$
\frac{\delta R(q)}{R}=\frac{\Delta R}{R} \frac{(1-q) / 2}{1+[A /(1-A)](1+q)}
$$

taking $\delta R(q=+1) \equiv 0$, as was specifically done by zeroshifting the measured $\delta R-\boldsymbol{H}_{\mathrm{a}}$ loops as described in Sec. III.B. Similar angular dependence of CPP-GMR was also recently measured in magnetic multilayers at $4.2 \mathrm{~K}$ [24].

Equation (10) may be exploited to determine $q=q_{\text {crit }}$ by using the set of $\sim 45$ triplets of $\left(I_{e}^{\text {crit }}, \boldsymbol{H}_{\mathrm{p}}^{\text {crit }},(\delta R / R)_{\text {crit }}\right)$ from the measurements of Sec. III.B, substituting $(\delta R / R)_{\text {crit }}$ into (10) (with $\Delta R / R \cong 0.87 \%$ ), and inverting to solve for $q_{\text {crit }}$. This yields $\sim 45$ discrete values for $I_{e}^{\text {crit }}(q)$, from which one obtains $F(q)$ using the right side of (7) (with $H_{\perp} \approx 7.5 \mathrm{kOe}$ [18]). The results of this procedure are plotted in Fig. 7.

Also included in Fig. 7 are results with $q_{\text {crit }}$ evaluated as

$$
\hat{\boldsymbol{m}}_{\text {ref }}=-\hat{z} \rightarrow q_{\text {crit }} \cong-\hat{z} \cdot \hat{\boldsymbol{H}}_{\mathrm{p}}^{\text {crit }}
$$

using (A2). However, (11) assumes circular pillars with negligible in-plane anisotropy, zero interlayer coupling, and complete spatial uniformity of $\hat{\boldsymbol{m}}_{\text {free }}$, none of which is assumed for (10). The use of (11) is expected to be most valid for critical points with the largest $\left|\boldsymbol{H}_{\mathrm{a}}\right|$, such as the larger $\left|q_{\text {crit }}\right|$ data extracted from Figs. 4,5. Fig.7 confirms that the results from (10) and (11) agree best for these data points. Using (10), the complete set of critical points (from $\delta R-H$ or PSD) in Fig. 7 appear to lie on a single curve.

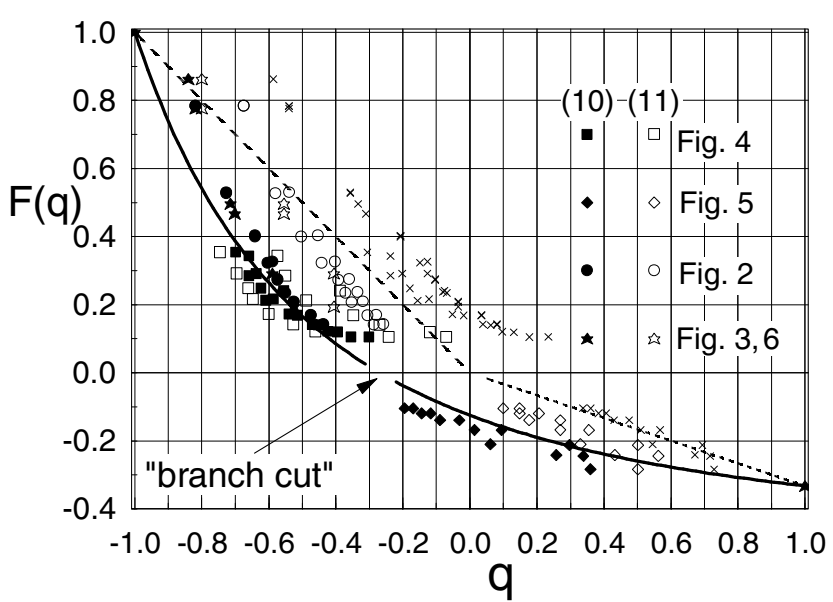

Fig.7. $F(q)$ using the data from Figs. 2-6 (as indicated), evaluated from the right side of (7), with $q$ from (10) (filled symbols) or (11) (open symbols). The smooth curves are generated from the left side of (7), with $\beta(q)$ from (9) (solid) or (12) (dashed). The points denoted with " $\times$ "are generated by evaluating $q$ using (10) with $A \equiv 0$.

Most noteworthy in Fig. 7 is the consistent agreement between the measured and predicted $q=\hat{\boldsymbol{m}}_{0} \cdot \hat{\boldsymbol{m}}_{\text {ref }}$ dependence when using (7) with $\beta(q)$ from (9), and using (10) (with $A=0.5$ ) to measure $q_{\text {crit }}$. (Deviations near $q \approx-0.8$ likely result from thermally reduced $I_{e}^{\text {crit }}$ for these lowest $\left|\boldsymbol{H}_{\mathrm{p}}\right|$ critical points from Figs. 2,3.) In particular, the predicted location of the "branch-cut" (see (8)) at $q \cong-0.27$ agrees remarkably well with the apparent absence of measurable critical points at/near the zero of $F(q)$. Its negative shift from $q=0$ results from the $d \beta / d q$ term first predicted in (6).

The dashed curve in Fig. 7 was computed from (7) using the following simple but discontinuous functional form for $\beta(q)$ :

$$
\beta^{ \pm}(q) / \beta(-1)=1 / 3-(2 / 3) \operatorname{sgn}(q)
$$

chosen to provide a rough approximation to the " $x$ " critical points that were extracted with the linear form of (10) (with $A \equiv 0$ ) universally used for $C I P$-GMR devices. The nonphysical discontinuity of this extracted $\beta(q)$ is further indicative of the physical connection between STT and MR

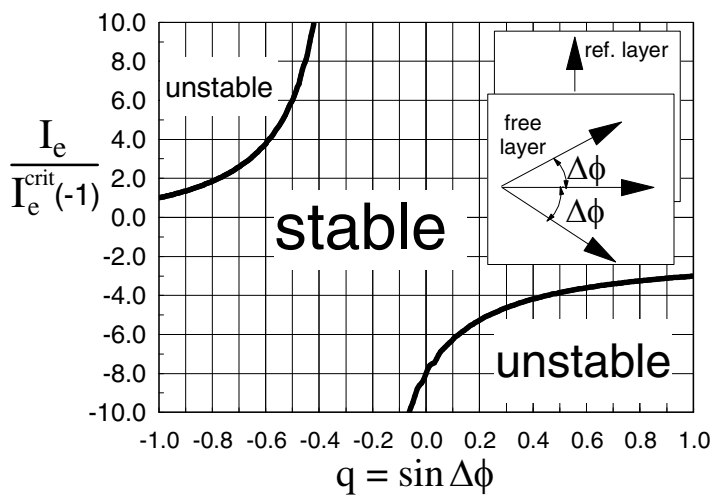

Fig. 8. $q-I_{e}$ stability space for the CPP-GMR read head configuration illustrated in the upper right, and computed as described in the text. 
in $C P P$ devices, as is implied by (9) and (10).

An important practical finding of this study is the heretofore unconsidered role of $d \beta / d q$ in governing the STT-induced instability in CPP-GMR read heads, as described here in (6). It can lead to some non-intuitive consequences regarding stable operational ranges of such devices. This is illustrated in Fig. 8, which delineates stability regions in $q-I_{e}$ space. The boundary curves are determined by solving (7) for $I_{e}^{\text {crit }}(q)$ in terms of the differential functional of $\beta(q)$, and then using $\beta(q)$ from (9) with $A=1 / 2$. (For simplicity, it is assumed here that $\left|\boldsymbol{H}_{\mathrm{p}}^{\text {crit }}\right| / H_{\perp} \ll<1$ and/or is slowly varying with $q$.)

For example, assuming a moderate head excitation $|q=\sin \Delta \phi| \leq 0.4$ about the bias point $\phi=0$, stable operation with STT present is predicted for bias currents satisfying $-4 \leq I_{e}^{\text {bias }} / I_{e}^{\text {crit }}(-1) \leq+10$. Hence, for stable operation with maximum $I_{e}^{\text {bias }}$ (and thus signal), a substantial 2.5-fold advantage is achieved by using positive $I_{e}^{\text {bias }}$, despite (since STT $\propto \beta)$ the fact that $\beta(q)$ increases monotonically with decreasing $q$, and that $q_{\text {crit }}\left(I_{e}>0\right)<q_{\text {crit }}\left(I_{e}<0\right)$. Due to the nonlinear $q$-dependence in (10), signal symmetry considerations may lead to other bias point choices.

\section{APPENDIX}

To first order in $\boldsymbol{m}^{\prime} \equiv\left(m_{x}^{\prime}, m_{y}^{\prime}\right), \hat{\boldsymbol{m}}=\overrightarrow{\mathfrak{R}} \cdot \hat{\boldsymbol{m}}^{\prime}$, with $m_{z}^{\prime}=1$, and $3 \times 3$ partitioned rotation matrix $\overrightarrow{\mathfrak{R}}=\left(\vec{R} \mid \hat{\boldsymbol{m}}_{0}\right)$ [25]:

$$
\hat{\boldsymbol{m}}_{0}=\left(\begin{array}{c}
\sin \theta \\
\cos \theta \sin \phi \\
\cos \theta \cos \phi
\end{array}\right), \vec{R}=\left(\begin{array}{cc}
\cos \theta & 0 \\
-\sin \theta \sin \phi & \cos \phi \\
-\sin \theta \cos \phi & -\sin \phi
\end{array}\right)
$$

Left-multiplying (1) by $\overrightarrow{\mathfrak{R}}^{-1} \equiv \overrightarrow{\mathfrak{R}}^{\top}$ yields, to $1^{\text {st }}$ order in $\boldsymbol{m}^{\prime}$ : $\left(\hat{z}^{\prime} \times d \hat{\boldsymbol{m}}^{\prime} / d t\right)+\alpha d \hat{\boldsymbol{m}}^{\prime} / d t=\gamma \overrightarrow{\mathfrak{R}}^{\top} \cdot\left[\boldsymbol{H}_{\mathrm{eff}}-\left(\boldsymbol{H}_{\mathrm{eff}} \cdot \hat{\boldsymbol{m}}\right)_{0} \overrightarrow{\mathfrak{R}} \cdot \hat{\boldsymbol{m}}^{\prime}\right]$. The $z^{\prime}$ component of this equation is null. Expressing $\boldsymbol{H}(\hat{\boldsymbol{m}})=\boldsymbol{H}\left(\hat{\boldsymbol{m}}_{0}\right)+\partial \boldsymbol{H} / \partial \hat{\boldsymbol{m}} \cdot\left(\hat{\boldsymbol{m}}-\hat{\boldsymbol{m}}_{0} \equiv \vec{R} \cdot \boldsymbol{m}^{\prime}\right)$ then yields (4).

For a free-layer with out-of-plane uniaxial anisotropy $H_{\perp}$, and a unidirectional in-plane field $\boldsymbol{H}_{\mathrm{p}}=\left(0, H_{\mathrm{p} y}, H_{\mathrm{p} z}\right)$, $E_{\text {free }} / M_{s} V_{\text {free }}=1 / 2 H_{\perp} m_{x}^{2}-H_{\mathrm{p} y} m_{y}-H_{\mathrm{p} z} m_{z}$. Assuming an in-plane $\hat{\boldsymbol{m}}_{\text {ref }}=(0, \sin \psi, \cos \psi)$, one can, using (3), (A1), and some matrix algebra, evaluate all quantities needed in (4).

The equilibrium $\vec{R}^{\top} \cdot \boldsymbol{H}_{\text {eff }}\left(\hat{\boldsymbol{m}}_{0}\right)=0$ approximately satisfies:

$$
\begin{gathered}
\phi \approx \tan ^{-1}\left(H_{\mathrm{p} y} / H_{\mathrm{p} z}\right) \\
\theta \approx \beta_{0} H_{\mathrm{st}} \sin (\phi-\psi) /\left(\left|\boldsymbol{H}_{\mathrm{p}}\right|+H_{\perp}\right)
\end{gathered}
$$

where $\beta_{0} \equiv \beta\left(\hat{\boldsymbol{m}}_{0} \cdot \hat{\boldsymbol{m}}_{\text {ref }}\right)$. In practical circumstances, $\theta<<1$. Approximating $\hat{\boldsymbol{m}}_{0} \cdot \boldsymbol{H}_{\mathrm{p}} \cong\left|\boldsymbol{H}_{\mathrm{p}}\right|$ from (A2), the $\overrightarrow{\boldsymbol{H}}^{\prime}$ tensor can be found using (3), (4), (A1), and some matrix algebra. The relevant quantities required for (5) are found to be:

$$
\begin{aligned}
& H_{x x}^{\prime}+H_{y y}^{\prime}=2\left|\boldsymbol{H}_{\mathrm{p}}\right|+\left(1-3 \sin ^{2} \theta\right) H_{\perp} \\
& H_{y x}^{\prime}-H_{x y}^{\prime}=\left[2 q \beta_{0}-\left(1-q^{2}\right) d \beta_{0} / d q\right] H_{\mathrm{st}}
\end{aligned}
$$

where $q \equiv \hat{\boldsymbol{m}}_{0} \cdot \hat{\boldsymbol{m}}_{\text {ref }}=\cos \theta \cos (\phi-\psi)$. The $d \beta / d q$ term in (A3) originates from the $\hat{\boldsymbol{m}}$-dependence of $\beta\left(\hat{\boldsymbol{m}} \cdot \hat{\boldsymbol{m}}_{\text {ref }}\right)$, and its contribution from (3) to $\partial \boldsymbol{H}_{\text {eff }} / \partial \hat{\boldsymbol{m}}$ in (4). Neglecting terms of order $\theta^{2}$ (see (A2)) then yields the result in (6).

\section{REFERENCES}

[1] J. Slonczewski,J. Magn. Magn. Mater., vol. 159, pp. L1-L7, 1996.

[2] L. Berger, Phys. Rev. B, vol. 54, pp. 9353-9358, 1996.

[3] M. D. Stiles and A. Zangwill, Phys. Rev. B, vol. 66, pp.014407-1-14, 2002

[4] S. Zhang, P.M. Levy, and A. Fert, Phys. Rev. Lett., vol. 88, pp. 236601$1-4,2002$.

[5] J. Slonczewski, J. Magn. Magn. Mater., pp. 324-338, 2002.

[6] J. Z. Sun, Phys. Rev. B, vol. 62, pp. 570-578, 2000.

[7] J. Grollier et al., Phys. Rev. B, vol. 67, pp. 174402-1-8, 2003.

[8] Z. Li and S. Zhang, Phys. Rev. B, vol. 69, pp. 134406-1-6, 2004

[9] D. M. Apalkov and P. B. Visscher, cond-mat/0405305, pp. 1-11 (2004)

[10] N. Smith, cond-mat/0406486, pp. 1-16, 2004. This prior work specifically excluded of the angle dependence of $\beta(q)$, and so used a different notation which included $\beta$ in the $H_{\text {st }}$ constant in (2).

[11] R. H. Koch, J. A. Katine, anf J. Z. Sun, Phys. Rev. Lett., vol. 92, pp. 088302-1-4, 2004.

[12] A. Fert et al., J. Magn. Magn. Mater., vol. 272-276, pp. 1706-1711 (2004).

[13] M. Tsoi et al., Phys. Rev. Lett., vol. 80, pp. 4281-4284 (1998).

[14] J. A. Katine, F. J. Albert, R. A. Buhrman, E. B. Myers, and D. C. Ralph, Phys. Rev. Lett., vol. 84, pp. 3149-3152, 2000.

[15] S. I. Kiselev et al., Nature, vol. 425, pp. 380-383, 2003.

[16] W. H. Rippard, M. R. Pufall, S. Kaka, S. E. Russek, and T. J. Silva, Phys. Rev. Lett., vol. 92, pp. 027201-1-4, 2004.

[17] I. N. Krivotorov et al., Phys. Rev. Lett., vol. 93, 166603-1-4, 2004.

[18] D. Lacour, J. A. Katine, N. Smith, M. J. Carey, and J. R. Childress, Appl. Phys. Lett., vol. 85, pp. 4681-4683, 2004.

[19] J. G. Zhu et al., IEEE Trans. Magn.., vol. 40, pp. 2323-2328, 2004.

[20] M. Covington, M. AlHajDarwish, Y. Ding, N. J. Gokemeijer, and M. A. Seigler, Phys. Rev. B, vol. 69, pp. 184406-1-8, 2004.

[21] F. B. Mancoff, R. W. Dave, N. D. Rizzo, T. C. Eschrich, B. N. Engel, and S. Tehrani, Appl. Phys. Lett., vol. 83, pp. 1596-1598, 2003.

[22] J. Xiao, A. Zangwill, and M.D. Stiles, Phys. Rev. B, vol. 70, pp.172405$1-4,2004$. This paper includes more general forms for $\beta(q)$ based on [5], that are not included (nor seemingly needed) here. The differences are expected to be small (M. Stiles, private communication).

[23] A. Kovalev, A. Brataas, and G. E. W. Bauer, Phys. Rev. B, vol. 66, pp. 224424-1-8, 2002; J. Manschot, A. Brataas, and G. E. Bauer, ibid, vol. 69, pp.092407-1-4, 2004

[24] S. Urazhdin, R. Loloee, and W. P. Pratt, Jr., Phys. Rev. B, vol. 71, pp. 100401-1-4, 2005.

[25] N. Smith, J. Appl. Phys., vol. 92, pp. 3877-3885, 2002. 